\title{
La realidad de convertirse en madre: vivencias de una adolescente ${ }^{1}$
}

\author{
Claudia Moreno Mojica² \\ Leidy Hernández Mancipe 3 \\ Tania Rincón Villamil4
}

doi:10.11144/Javeriana.ie19-2.rcmv

Cómo citar: Moreno Mojica C, Hernández Mancipe L, Rincón Villamil T. La realidad de convertirse en madre: vivencias de una adolescente. Investig Enferm Imagen Desarr. 2017;19(2):13-28. http://dx.doi.org/10.11144/Javeriana.ie19-2.rcmv

1. Artículo derivado de investigación. Fecha de recepción: 29 de septiembre de 2015. Fecha de aceptación: 29 de febrero de 2016.

2. Enfermera, Universidad Pedagógica y Tecnológica de Colombia (UPTC), Tunja, Colombia. Magíster en Enfermería, Universidad de La Sabana, Chía, Colombia. Candidata a Magíster en Pedagogía, Universidad Santo Tomás, Bogotá, Colombia. Docente de la Escuela de Enfermería, UPTC. Correo electrónico: claudiamaria.moreno@uptc.edu.com

3. Estudiante de pregrado en Enfermería, Universidad Pedagógica y Tecnológica de Colombia (UPTC), Tunja, Colombia. Miembro del semillero del grupo de investigación Calidad y Cuidado en Enfermeria. Correo electrónico: leidyhernadez0202@gmail.com

4. Estudiante de pregrado en Enfermería, Universidad Pedagógica y Tecnológica de Colombia (UPTC), Tunja, Colombia. Miembro del semillero del grupo de investigación Calidad y Cuidado en Enfermería. Correo electrónico: taniarincon2609@gmail.com 


\section{Resumen}

Introducción: En el mundo, el embarazo durante la adolescencia se considera un problema de salud pública, y uno de los ámbitos que influyen en esta situación es el psicosocial, en el cual la adolescente se enfrenta a la aceptación o rechazo por parte de su familia y contexto social inmediato, que la lleva a tomar la decisión de adoptar o no el rol materno. Objetivo: Conocer y comprender, mediante la técnica del relato biográfico, el proceso de convertirse en madre adolescente. Métodos: Investigación cualitativa de tipo descriptivo, con diseño de relato biográfico, que se utiliza para describir exhaustivamente la experiencia vivida por una persona. Resultados: Se obtuvieron ocho categorias: "recordando mi niñez", "mi primer amor", "lo prohibido lo más deseado", "lo inesperado llegó", "enfrentando la dura realidad", "aprendiendo a ser mamá", "adopción del rol materno" y "en busca de mis sueños". Conclusiones: Las categorías generadas y analizadas a la luz de la literatura y la teoría de Mercer permitieron conocer y comprender el proceso por el cual transita la adolescente para convertirse en madre, desde donde si bien el aspecto fisiológico en un factor de riesgo determinante durante la gestación, son los aspectos psicológicos, sociales y del entorno inmediato los que determinan la calidad de vida de la díada y, por ende, el cuidado integral, holístico y humano que el equipo de salud debe brindar.

Palabras clave: embarazo; salud del adolescente; enfermería

\section{The Reality of Becoming a Mother: Experiences of a Teenager}

\section{Abstract}

Introduction: In the world, teenage pregnancy is considered a public health problem and one of the areas that influence this situation is psychosocial, as the teenager faces the acceptance or rejection by family and immediate social context, which leads her to make the decision to adopt or not the maternal role. Objective: To know and understand, through the technique of biographical narrative, the process of becoming a teenage mother. Methods: Qualitative descriptive research, with a biographical account design, which is used to comprehensively describe the experience of a person. Results: eight categories emerged, "childhood memories", "my first love", "the forbidden but most desired", " facing the unexpected", "facing the harsh reality", "learning to be a mom," "adopting the maternal role" and "pursuing my dreams". Conclusions: The categories generated and analyzed in the light of literature and the theory of Mercer allowed to know and understand the process a teenager goes through when becoming a mother, where although the physiological aspect is a risk factor during pregnancy, the psychological, social and the immediate environment are the aspects that determine the quality of life of the dyad and, therefore, the comprehensive, holistic and human care that healthcare professionals should provide.

Keywords: pregnancy; adolescent health; Nursing 


\section{A realidade de se tornar uma mãe: vivencias de uma adolescente}

\section{Resumo}

Introdução: No mundo, a gravidez na adolescência é considerada um problema de saúde pública e uma das áreas influenciando tal situação é a psicossocial, quando a adolescente defronta a aceitação ou rejeição pela família e pelo contexto social imediato, levando ela à decisão de adotar ou não o papel maternal. Objetivo: Conhecer e compreender, através da técnica de narrativa biográfica, o processo de se tornar uma mãe adolescente. Métodos: pesquisa qualitativa descritiva, com design de relato biográfico, que é usado para descrever completamente a experiência de uma pessoa. Resultados: oito categorias foram obtidas: "lembrando-me da minha meninice", "meu primeiro amor", "o proibido é o mais desejado", "chegou o inesperado", "arrostar a realidade dura", "aprendendo a ser mãe", "adoção do papel materno" e "na procura dos meus sonhos". Conclusões: As categorias geradas e analisadas à luz da literatura e da teoria de Mercer permitiram conhecer e entender o processo pelo qual passa a adolescente para se tornar uma mãe, onde, embora o aspecto fisiológico, é fator de risco significativo para a gestação, é o aspecto psicológico, social e do ambiente imediato que determinam a qualidade de vida da díade e, portanto, o cuidado abrangente, holístico e humano que os profissionais de saúde devem fornecer.

Palavras-chave: gravidez; saúde do adolescente; enfermagem 


\section{Introducción}

Según la Organización Mundial de la Salud, la adolescencia es el periodo comprendido entre los 10 y los 19 años de edad, durante el cual el individuo adquiere la capacidad reproductiva, transita por los patrones psicológicos de la niñez a la adultez y consolida la independencia socioeconómica (1). Este transitar se ve influenciado por la cultura, las características de la familia, las condiciones socioeconómicas y el nivel educativo, que pueden ser factores protectores o de riesgo frente a la incidencia del embarazo y maternidad en este grupo heterogéneo y vulnerable (2).

Así es como el embarazo durante la adolescencia se considera un problema mundial de salud pública, por el incremento de la morbilidad y mortalidad materna y neonatal (3). Según Barón (4), el mayor riesgo del embarazo adolescente no parece estar influenciado por factores fisiológicos, sino por variables socioculturales y condiciones de cuidado y apoyo familiar, por lo que se encuentran dos grupo de adolescentes: aquellas que planean la gestación por salir de su hogar en busca de afecto y como finalización de la violencia a la que son sometidas y aquellas que no lo planearon y que, junto a actitudes de negación generan, crisis en la nueva madre y su familia (5-7).

Un sentimiento presente en las adolescentes durante la gestación es verse privadas de las actividades que pertenecen a su edad, al asumir el rol de adultas y madres en una etapa en la cual aún no ha desarrollado su identidad personal. Según Climent (8), este sentimiento se logra transformar a medida que la gestante resignifica el ser madre como un proceso que se desarrolla en el tiempo e incluye la gestación, el parto y el posparto, así como el proyecto de vida donde trasciende lo referido al hijo y la pareja e incluye la posibilidad de continuar con estudios y realización de trabajos (9).

El convertirse en madre adolescente tiene distintos significados según el estilo de vida y aceptación de este, situación que reclama una atención especial por parte de los actores de la sociedad, las familias, los establecimientos educativos, los entes políticos-territoriales y las entidades de salud. Por ello es necesario conocer y comprender, mediante la técnica del relato biográfico, el proceso de convertirse en madre adolescente, tras participar en el 2014 en el programa Creciendo Juntos: Activación del Rol Materno durante el Control Parental. Este sirvió de base de evaluación, mejoramiento y construcción de herramientas para la planeación del cuidado que brinda el equipo interdisciplinario a la gestante, o compañero, cuyo fin es disminuir la morbimortalidad materna.

\section{Métodos}

Investigación cualitativa de tipo descriptivo, con diseño de relato biográfico, que se utiliza para describir exhaustivamente la experiencia vivida por una sola persona, cuyo objetivo es ampliar el grado de comprensión de la realidad, basándose en las percepciones, las expectativas, las emociones y las 
opiniones de los actores que se encuentran implicados en los procesos de salud-enfermedad (10).

De acuerdo con Mallimaci y Giménez (11), el método biográfico trabaja analiticamente sobre la narrativa de una persona acerca de sí misma y su relación con el fenómeno que se pretende estudiar. En este tipo de estudios se busca producir nuevos conocimientos que permitan comprender el significado vivido por el protagonista, para brindar cuidado a seres unitarios con responsabilidad y coherencia (12).

En este artículo se traen a colación las vivencias de una adolescente de 17 años de edad, quien describe su experiencia frente a dos gestaciones a temprana edad y refiere cómo los factores familiares, culturales, escolares, e intervenciones de enfermería influyeron en el proceso para convertirse en madre, desde su activación y transición por la gestación y parto hasta el posparto, cuando el establecimiento de comportamientos y responsabilidad propias le permitieron alcanzar un nuevo concepto e identidad de sí misma como madre.

La técnica de recolección es la entrevista cualitativa o en profundidad, la cual se registró por medio de grabaciones y se transcribió textualmente, previa firma del consentimiento informado, concertado en dos citas con la adolecente, donde se garantizó privacidad y no hubo interferencia de otros actores. La entrevista se realizó a través de dos encuentros con un tiempo estimado de una hora. El método de análisis empleado fue en el enfoque de análisis de contenido de textos biográficos, que toma como referencia la metodología de Taylor-Bogdan (13).

En el desarrollo de este estudio se siguieron los lineamientos de la normatividad ética de investigación propuesta en Colombia mediante la Resolución 008430 de 1993, por la cual se establecen las normas académicas, técnicas y administrativas para la investigación en salud. También se tuvieron presente las consideraciones internacionales en el uso del consentimiento informado (artículo 15) y participación voluntaria (14), sin vulnerar ninguno de los tres principios universales: respeto por las personas, beneficencia y justicia, contemplados en las pautas éticas internacionales para la investigación biomédica en seres humanos, establecidas por el Consejo de Organizaciones Internacionales de las Ciencias Médicas, en colaboración con la Organización Mundial de la Salud. Por ello se presenta el relato biográfico con nombres ficticios como derecho a la privacidad $(14,15)$.

\section{Resultados y discusión}

El aspecto central de la investigación se da a través de la comprensión del proceso de convertirse en madre adolescente, el significado de los cambios, los sentimientos, las actitudes, las emociones y la interpretación que hace la madre de la situación en relación con las fases por las que ha transitado para alcanzar su nuevo rol (16). Los temas centrales dieron origen a ocho categorias (figura 1). 


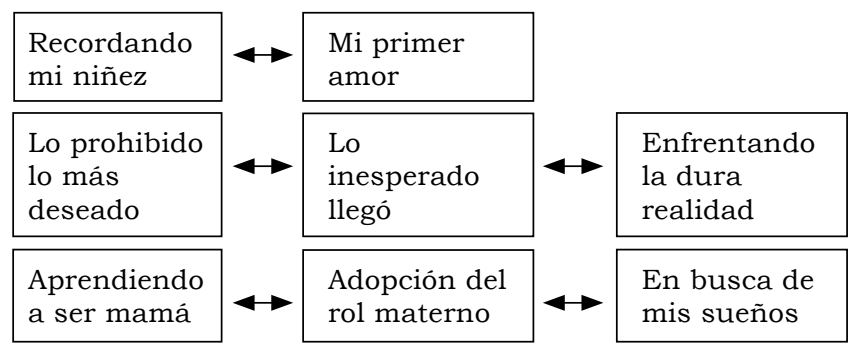

Figura 1. Categorias de los temas centrales de investigación

Fuente: elaboración propia.

Al realizar la triangulación con planteamientos teóricos, surge la relación entre las categorías "Recordando mi niñez "y "Mi primer amor", donde, según Climent (8), los estilos parentales determinan los comportamientos que los hijos establecen en el futuro. Así, adolescentes que tienen padres que no les demuestran su afecto son hostiles, no receptivos y autoritarios, presentan embarazos a temprana edad como resultado de una situación conflictiva donde manifiestan tristeza y rencor frente a la inestabilidad familiar y esperan ser compendiadas y amadas por su pareja.

\section{Recordando mi niñez}

"Mi nombre es Juliana, tengo 17 años y dos hijos, de dos y nueve meses de edad. Desde muy pequeña siempre me destaqué por ser responsable con mi estudio y tener buen comportamiento, tenía muchos amigos, que siempre fueron mi apoyo en los momentos difíciles. En mi casa vivía con mi mamá, mi papá y mi hermana mayor. Allí todo era diferente, fui criada en un hogar con muchas restricciones y pocos valores; mi padre maltrataba físicamente a mi madre, además de esto la insultaba con palabras vulgares. Tuve que observar cómo mi padre imponía su autoridad sobre nosotras de una forma machista, al pensar que por ser mujeres éramos más vulnerables y nos podía tratar como se le diera gana".

"Nunca les importé a mis padres, o al menos eso pienso. Me acuerdo tanto que el día de la entrega de boletines del colegio comenzaron a discutir y no porque hubiera reprobado alguna materia, sino porque ninguno quería asistir, ninguno de los dos tenía tiempo para mí. Ese día lloré mucho, porque sentí que no valía nada. Ellos tenían preocupaciones tal vez más importantes que yo".

\section{Mi primer amor}

Situaciones como las vividas por esta adolescente reflejan la carencia afectiva, entendida como la necesidad de compañia, incluye el amor romántico como medio para obtener afecto, comprensión y otorgar sentido a la vida (17). 
"Cuando más triste me encontraba, conocí a Camilo. Cómo describirlo, era el niño más guapo y dulce del salón, podría decir que del colegio entero. Él estaba en un curso más adelante, mientras yo estaba en octavo con apenas 14 años. Empezó a conquistarme por medio de cartas, saludos y, de vez en cuando, me enviaba dulces con un compañero. Con el paso del tiempo y de muchos chocolates, empecé a sentir que él me comprendía, que entendía todos mis problemas y las situaciones que vivia en mi casa. Realmente se convirtió en mi refugio, cada palabra, cada gesto y cada mirada hicieron que me enamorara de él”.

"Cuando llegaba a mi casa, con tantas inquietudes y curiosidades dando vueltas en mi cabeza, sentía la necesidad de confesarle todo lo que sentía a mi madre, de preguntarle, por qué me estaba pasando esto a mí; pero lo único que escuchaba de ella, eran gritos y reproches. Siempre andaba de mal genio, muchas veces por el trabajo, por las peleas con mi papá, por la falta de plata en la casa; así que nunca me nació contarle nada, pensé que de pronto era un problema más para ella. En realidad, nunca encontré un apoyo en nadie de mi familia”.

En lo referente a las categorias "Lo prohibido lo más deseado", "Lo inesperado llegó" y "Enfrentado la dura realidad", se observa una correlación donde el sentimiento emocional que se genera en los adolescentes estimula emociones caracterizadas por idealización, atracción sexual y contacto físico, que los lleva a dar inicio a una sexualidad activa sin protección desde la omnipotencia e incredulidad, como conducta irresponsable que los expone a embarazos no deseados $(7,17,18)$.

\section{Lo prohibido, lo más deseado}

"Cada día me sentía más atraída y más enamorada de Camilo, hasta que una tarde, la pasión y los sentimientos nos llevaron a tener nuestra primera vez juntos. Fue algo bonito y muy especial para mí, y después de este encuentro vinieron muchas más tardes de amor y locura. Llevábamos tres meses de noviazgo, nunca nos cuidamos, a pesar de que en el colegio teníamos clases de salud sexual; pero en un momento de furor nunca pensamos en un embarazo o en una enfermedad, solo queríamos vivir el momento, sin importar las consecuencias".

\section{Lo inesperado llegó}

Gómez y colaboradores (19) y Álvarez y colaboradores (20) concluyeron que las adolescentes, al enterarse del estado de gestación, transitan por emociones de miedo y duda frente a la reacción de su familia y pareja. Algunas de ellas piensan en algún momento en abortar a su hijo por miedo al castigo y rechazo social al que deben enfrentarse. La toma de esta decisión está relacionada con aspectos morales, religiosos y culturales, que llevan a la joven a decidirse por este procedimiento, como salida rápida a la situación 
o afrontar el paso a la adultez y maternidad, y continuar el embarazo como medida de protección de una nueva vida $(19,20)$.

"Mi cuerpo empezó a cambiar sin darme cuenta. Mi periodo menstrual se retrasó. Aumenté de peso. Lloraba por todo. Me ponía brava por cualquier cosa. Tenía náuseas, mareos constantes. Llegué a pensar que estaba enferma. Le conté a Camilo lo que estaba pasando, él me dijo sorprendido "No puede ser, ¿estás embarazada?". Yo no lo podía creer, y ese mismo día, al salir de clases, compramos una prueba de embarazo, de esas que venden en la droguería. Me dio tanta pena y, al mismo tiempo, tanto miedo de pensar que yo podía estar embarazada".

"Llegué a mi casa desesperada, quería hacerme la prueba de una vez por todas, pero no pude porque mi mamá y mi hermana estaban en la casa. Finalmente, me hice la prueba el día siguiente. No tenía ni idea de cómo se realizaba esa prueba. Duré mucho tiempo encerrada en el baño. Mi mamá golpeaba la puerta, y me decía: 'Juliana, ¿cuál es la demora en ese baño, no ve que se nos va a hacer tarde a todos'. Cuando me di cuenta de que el resultado era positivo, sentí como mi corazón latía tan rápido y tan fuerte como si se me fuera a salir del pecho y todo mi mundo se derrumbó en ese momento".

\section{Enfrentando la dura realidad}

Para Noguera y Alvarado (2), el embarazo en la adolescente genera un impacto psicológico, social y cultural que se denomina sindrome de las embarazadas adolescentes, que comprende la ambivalencia entre la identidad que se está creando como joven frente a la identidad materna y conductas de mujer adulta, inicio laboral para sustento de la constitución de una familia estable; de igual forma, se presenta el fracaso ante la adquisición de independencia, abandono escolar, limitación en el proyecto de vida y falta de apoyo familiar y de la pareja.

"Entrando al colegio hablé con Camilo, y llorando le conté todo. No sabíamos qué hacer. Éramos un par de niños, con otro niño en camino. Tuvimos pensamientos de perder el bebé, de no tenerlo; pero dejamos que la cabeza se enfriara y decidimos continuar con todo esto y enfrentar a toda costa lo que se viniera. Lo más angustiante fue pensar cómo decirles a mis padres; sobre todo a mi papá. Ya me imaginaba sus gritos de reproche y decepción. Lloré muchas noches, no quería comer, no quería hablar con nadie, hasta que un día mi mamá me preguntó 'Juliana, ¿usted está embarazada o qué es lo que tiene?, usted anda muy rara, hace cuánto es que no le llega la regla', y me dijo 'vamos a la droguería a comprar una prueba de embarazo', yo comencé a llorar y le respondi 'Mami, no gaste su plata, estoy embarazada".

El papel de la familia es considerado fundamental en la forma como es vivido el proceso de embarazo en la adolescencia, de la que se espera apoyo, comunicación empática y soporte afectivo y emocional. No obstante, las relaciones, los contextos y los tipos de familia en las que crecen estas adolescentes no siempre representan un factor protector durante el acompañamiento y soporte de la gestación, ya que frente a este proceso existen familias que 
irrespetan, ignoran y abandonan sus hijas, lo cual acentúa la falta de apoyo, protección y seguridad que brindaban ante la gestación, aparte de que genera una ruptura entre el vínculo de padres, entorno social e hija, que las lleva a sentir vergüenza y rechazo, por lo que la deserción escolar y salida del núcleo familiar es una de las consecuencias y factores de riesgo a largo plazo en la calidad de vida de la díada $(21,22)$.

"Mi mamá comenzó a llorar. No lo podía creer. No era lo que quería para mí, y ahora venía lo más difícil: contarle a mi papá, ¿cómo iba a reaccionar?, ¿qué me iba a decir?, eran preguntas sin respuestas que atormentaban mi cabeza. Al final, mi madre le confesó todo a mi padre. Yo no tuve el valor de enfrentarlo, ni mucho menos Camilo. Qué gran decepción se llevó él. Desde entonces no volvió a hablarme. Muchas veces intenté acercarme, pero siempre recibí rechazo y más sermones. Poco a poco mi barriguita fue creciendo. El rechazo no solo fue por parte de mi padre, en el colegio, mis compañeras me miraban con lástima; me sentía mal, no quería volver a clases, mis notas fueron de las peores ese periodo, así que mi papá me retiró del colegio, abandonando mis estudios, mis amigos, mis maestros; todo lo que había significado algo para mî".

\section{Aprendiendo a ser mamá adolescente}

Esta categoría determina que aun cuando muchos de estos embarazos no fueron deseados, las jóvenes lo asumen desde la incertidumbre, la soledad, el temor, la angustia y el miedo que genera el proceso de gestación, y los cuidados propios y de su hijo (5). De igual forma, expresan sentimientos placenteros como amor o ternura, y desplacenteros, como exigencia, cansancio y agobio, al estar a cargo del cuidado de su recién nacido. Algunas adolescentes sienten la necesidad de continuar con sus estudios, y quienes los retoman experimentan conflicto entre sus necesidades y las de sus hijos. Ante esta situación, la madre de la adolescente asume el rol de cuidadora, siendo esta su principal red de apoyo $(23,24)$.

"Camilo abandonó también el colegio. Tenía que hacerse responsable, y ya no pensar en dos sino en tres. Pasamos de ser niños a ser adultos en una abrir y cerrar de ojos. Él comenzó a trabajar en un parqueadero; con su sueldo pudimos ahorrar para comprar algunas cosas del bebé; pasó el tiempo y llegó el día del nacimiento de nuestro hijo. Fue muy difícil para mí aprender todos los cuidados que requiere un bebé, como darle de comer, saber en qué momento hay que cambiarlo, bañarlo, aunque las enfermeras del hospital me enseñaron muchas cosas, se me hacía muy difícil hacerlas sola".

"Camilo me colaboraba en lo que podía; pero aún no vivíamos juntos, por la falta de dinero. Mi mamá me ayudaba en la casa, y aunque nunca me lo imagine, mi hermana se convirtió en un gran apoyo. Por el contrario, la relación con mi papá era cada vez peor: me insultaba, me hacía sentir mal y el llanto de mi hijo le molestaba. Al mes de nacido, empecé a estudiar en Paipa, en un instituto de validación. Desde ahí, mi hijo y yo nos separamos, pues era mi mamá quien cuidaba de él. Me iba muy temprano a estudiar y 
cuando llegaba a la casa ya estaba dormido, y así todos los días hasta que pude graduarme de bachiller. Asi fuera muy duro, todo este esfuerzo lo hacía por él, para que algún día se sintiera orgulloso de mí".

La madre adolescente enfrenta el cambio de vida de manera abrupta, asumiendo la responsabilidad de conformar y mantener un hogar donde la pareja asume el rol laboral con ingresos bajos, que no les permiten mantener la independencia para satisfacer sus necesidades. Sumado a esto, la joven se expone a situaciones de violencia social y familiar, al no poder reclamar sus derechos por miedo al fracaso en su nueva etapa de vida, aspectos que se transforman en potenciales riesgos psicobiológicos para la diada, al depender económica y emocionalmente de terceros. En relación con este aspecto, se presenta el aumento en la probabilidad en nuevos de embarazos, como repetición de un patrón desadaptativo, que no cuenta con factores protectores que influyan en el reconstrucción del proyecto de vida de la adolescente (25).

"Pasado el tiempo, Camilo logró conseguir un trabajo más estable; vendía comidas rápidas en el centro de la ciudad. Con el sueldo que ganaba, nos fuimos a vivir los tres en arriendo. La convivencia, inicialmente, fue muy dura, pues Camilo llegaba tomado a la casa, me reprochaba, me insultaba, golpeaba las pocas cosas que teníamos, incluso un día llegó a maltratarme físicamente. Sufrí mucho, no queria repetir la misma historia de mi mamá, pero tampoco quería dejar a mi hijo sin un padre; y decidí aguantarme lo que fuera, no quería volver a mi casa derrotada, quería demostrar que todo estaba bien".

Por último, se encuentran las categorias "Adopción del rol materno" y "En busca de mis sueños", en las cuales se confronta la teoría expuesta por Ramona Mercer, base de construcción del programa Creciendo Juntos, donde el convertirse en madre se define como un proceso interactivo de desarrollo y transición que se produce a lo largo del tiempo, en el que la madre crea un vínculo con el hijo, aprende las tareas de cuidado del rol y expresa placer y gratificación con este $(25,26)$.

Para Morillo (23), el vínculo de la adolescente con su hijo es un proceso que comienza antes del nacimiento, en que influye la historia personal de la mujer y también en el contexto social que la constituye, por lo que intervenciones de enfermería que promuevan el apoyo emocional, informativo, físico y de valoración donde la madre o la pareja participen permiten la transición a la maternidad desde la activación del rol y el convertirse en madre.

\section{Adopción del rol materno}

Según Meleise (27), Mercer estableció, a partir de investigaciones cualitativas, fases o estadios que conducen el proceso de convertirse en madre, por las cuales, desde las vivencias expuestas en el relato biográfico, ha transitado la adolescente de la siguiente forma: fase de compromiso y preparación, que se presenta durante la gestación e incluye los primeros ajustes sociales y psicológicos del embarazo, donde las madres aprenden las expectativas del rol, fantasea sobre este. 
"Cuando mi primer hijo tenía seis meses de edad, quedé en embrazo nuevamente. No entiendo qué sucedió: llevaba las cuentas claras. Otra vez tuve miedo, era una responsabilidad más grande, pues ya no era un bebé, sino que ahora eran dos. Cuando le conté a mi familia, creo que nuevamente se decepcionaron de mí; pero no me importó, Camilo y yo seguimos enfrentando este embarazo, pensamos que había sido un regalo de Dios, por eso lo aceptamos con mucho amor".

"Permanecí en mi casa todo el embarazo. Asistí a mis controles prenatales en el Centro de Salud. Participé con Camilo en una jornada de enfermería para jóvenes en embarazo. Allí nos enseñaron a cuidar a nuestro bebé, aprendimos a bañarlo, a cambiarlo, a darle sol, a limpiarle el ombligo, a darle de comer, a darle amor y protección. Gracias a ese taller, pude expresar lo que sentía y aprender muchas cosas de las que no tenía idea, ni siquiera con la llegada de mi primer hijo; y que fueron de gran ayuda para para todo el proceso mi gestación y mi proyecto de vida".

"Cuando nos enteramos de que era una niña, nos sentimos felices, porque íbamos a tener la parejita. Camilo trabajó con dedicación, ahorró dinero y compramos todo lo que necesitábamos para el cuidado de nuestra niña. Esta vez me sentía más segura de cuidar a mi bebé".

La fase de consecución, práctica y recuperación física que plantea Mercer empieza cuando el niño nace e incluye el aprendizaje del rol: "Entonces llegó mi niña, la luz de mis ojos; con ella pude crear un vínculo más fuerte; tal vez más que con mi primer hijo. No es que al niño no lo quiera, pero he podido compartir más sentimientos con mi hija. Las cosas con Camilo han mejorado, ha sido más responsable, más amoroso, aunque como todas las parejas tenemos dificultades y atravesamos por momentos difíciles, quiero ser la mejor mamá para mis hijos. No quiero que a mis hijos les suceda lo mismo, por no tener un padre y una madre en quien apoyarse, con quien hablar, a quien expresarle sus sentimientos, sus miedos y sus dudas".

Finalmente, en la fase de normalización e integración de la identidad maternal, la madre hace que el nuevo rol encaje en su estilo de vida, basándose en experiencias pasadas. La mujer aquí experimenta un sentimiento de armonía y confianza en el modo que lleva a cabo el nuevo rol y restablece su proyecto de vida a parir del apoyo social y educativo brindado (28).

"Ahora mi hijo está en un jardín y yo cuido a mi niña todo el tiempo. Es mi consentida. Desde que ella llegó a nuestras vidas ha cambiado todo, incluso la relación con mi padre. Mi hermana adora a sus sobrinos y cuento con todo el apoyo de mi suegra que también ha sido una gran ayuda".

\section{En busca de mis sueños}

"No es nada fácil tener hijos siendo adolescente, ya que tenemos que privarnos de muchas cosas, de amigos, de fiestas, de conocer nuevas personas, nuevos lugares. Si yo pudiera devolver el tiempo, pensaría con más calma y haria mejor las cosas. No cambio a mis hijos por nada. Ellos son mi mayor bendición, y en este momento pienso que no he truncado mis sueños, solo 
he retrasado mi proyecto de vida, porque quiero seguir estudiando, quiero ser la pediatra que siempre soñé".

"Convertirme en madre adolescente ha sido una experiencia muy dura. A todas aquellas niñas que en este momento están pasando por una situación similar a la mía, les digo que piensen muy bien las cosas, que para todo hay tiempo. Lo primordial es perseguir los sueños, estudiar, tener una estabilidad económica, tener una profesión y, de esta manera, no depender de nadie. A las niñas que ya tienen a sus bebés como yo, solo me queda decirles que la vida sigue, y aunque sea muy difícil afrontar este nuevo rol de ser madre, sigamos en busca de nuestros sueños, que nuestros hijos sean el motivo para lograr y alcanzar todas las metas que nos proponemos".

\section{Conclusiones}

Las vivencias expuestas en el relato biográfico determinan cómo estrategias desarrolladas por enfermería, que promueven la salud de la mujer, permiten individualizar el cuidado y son las más eficaces en el proceso de convertirse en madre. Esto ocurre durante la enseñanza transmitida en el estadio de consecución, práctica y recuperación física, como primera fase para la activar las expectativas del rol y generar el vínculo o unión entre la díada (29).

Las categorias generadas y analizadas a la luz de la literatura y la teoría de Mercer permitieron conocer y comprender el proceso por el cual transita la adolescente para convertirse en madre, desde donde si bien el aspecto fisiológico en un factor de riesgo determinante durante la gestación, son los aspectos psicológicos, sociales y del entorno inmediato los que determinan la calidad de vida de la díada y, por ende, el cuidado integral, holístico y humano que el equipo de salud debe brindar (30).

El programa Creciendo Juntos: Convertirse en Madre durante la Adolescencia retomará las categorías para estructurar las siguientes dos estrategias: Visita Domiciliaria a la Gestante Adolescente, Pareja o Familia, por parte del equipo interdisciplinario: enfermera, médico y psicólogo; de igual forma, se plantea el estudio de caso, donde se socializará la situación clínica y la narrativa de enfermería, con el fin de dar a conocer a las entidades de primer nivel la realidad de la adolescente gestante que hace parte del programa, a efectos de construir intervenciones de cuidado integral que lleven a disminuir la morbimortalidad y a crear factores protectores que abolen conductas repetitivas y mejoren la calidad de vida.

\section{Financiación}

Ninguna.

\section{Conflictos de interẽs}

Ninguno.

\section{Agradecimientos}

Al Grupo de Investigación Calidad y Cuidado en Enfermería (Escuela de 24 Enfermería) de la Universidad Pedagógica y Tecnológica de Colombia y al Hospital Regional de Duitama. 


\section{Referencias}

1. Quesada M, Romero M, Prieto M, Rodríguez C. Caracterización social del embarazo en la adolescencia. Archivo Médico de Camagüey [internet]. 2010 [citado 2015 mar];14(3). Disponible en: http://scielo.sld.cu/ scielo.php?script=sci_arttext\&pid=S1025-02552010000300010\&lng=es

2. Noguera N, Alvarado H. Embarazo en adolescentes: una mirada desde el cuidado de enfermería. Revista Colombiana de Enfermería [internet]. 2010 [citado 2015 abr];7:151-60 Disponible en: http://www.uelbosque.edu.co/sites/default/files/publicaciones/revistas/revista_colombiana_enfermeria/volumen7/embarazo.pdf

3. Domínguez R, Herazo Y, Beltrán, M. Edad de la gestante adolescente como factor de riesgo para complicaciones en el embarazo. Cartagena (Colombia) 2009. Rev Colomb Obstet Ginecol [internet]. 2011 [citado 2015 mar];62(2):141-7. Disponible en: http://www.fecolsog.org/userfiles/file/revista/Revista_Vo162No2_Abril_Junio_2011/v62n2a04.pdf

4. Barón M. Embarazo en adolescentes: un problema de salud pública [internet]. 2011. Disponible en: http://www.ellider.com.co/2011/02/15/ embarazo-en-adolescentes-un-problema-de-salud-publica/

5. Prías H, Miranda C. Experiencias de adolescentes embarazadas en control prenatal. Aquichán [internet]. 2009 [citado 2015 may];9(1):93-105. Disponible en: http://www.redalyc.org/articulo.oa?id=74111465009

6. Salinas S, Castro M, Fernández C. Vivencias y relatos sobre el embarazo en adolescentes: una aproximación a los factores culturales, sociales y emocionales a partir de un estudio en seis países de la región [internet]. Ciudad de Panamá: Unicef; 2014. Disponible en: http://www.unicef.org/ecuador/embarazo_adolescente_5_0-2bis.pdf

7. Dalia C, Rojas P, Alarcón M, Calderón B. Vivencia del embarazo en adolescentes en una institución de protección en Colombia. Index Enferm [internet]. 2010 [citado 2015 abr];19(2-3). Disponible en: http://scielo. isciii.es/scielo.php?script=sci_arttext\&pid=S1132-12962010000200012

8. Climent G. Voces, silencios y gritos: los significados del embarazo en la adolescencia y los estilos parentales educativos. Revista Argentina de Sociología [internet]. 2009 [citado 2015 may];(12-13):186-213. Disponible en: http://www.scielo.org.ar/pdf/ras/v7n13/v7n13a04.pdf

9. Colmenares Z, Montero L, Reina R, González Z. Intervención de enfermería durante la clínica prenatal y conocimiento sobre riesgos fisiológicos de la adolescente embarazada. Enfermería Global [internet]. 2010 [citado 2015 may];(18). Disponible en: http://revistas.um.es/ eglobal/article/view/93631

10. Ortiz L. Curso investigación cualitativa [internet]. [Citado 2015 mar]. Disponible en: http://datateca.unad.edu.co/contenidos/401122/ Contenido\%20para\%20descarga/MODULO

11. Mallimaci F, Giménez B. Historia de vida y método biográfico. En: Estrategias de investigación cualitativa [internet]. Barcelona: Gedisa; 2006. Disponible en: http://jbposgrado.org/icuali/M\%E9todo\%20 biogr\%E1fico.pdf 
12. Muñoz Begoña M, Gálvez González M, Amezcua M. Cómo estructurar y redactar un relato biográfico para publicación. Index Enferm [internet]. 2013 [citado 2015 dic];22(1-2):83-8. doi: http://dx.doi.org/10.4321/ S1132-12962013000100018

13. Taylor SJ, Bogdan R. Introducción a los métodos cualitativos de investigación: la presentación de los hallazgos. Barcelona: Paidós; 1998.

14. Resolución 008430/1993, por la cual se establecen las normas científicas, técnicas y administrativas para la administración en salud [internet]. Ministerio de Salud de Colombia. Disponible en: https:// www.unisabana.edu.co/fileadmin/Documentos/Investigacion/comite_de_etica/Res__8430_1993_-_Salud.pdf

15. Stepke F. Pautas éticas internacionales para la investigación biomédica en seres humanos [internet]. Ginebra: Consejo de Organizaciones Internacionales de las Ciencias Médicas (CIOMS) y Organización Mundial de la Salud (OMS); 2002. Disponible en: http://www.paho. org/Spanish/BIO/CIOMS.pdf

16. Villamizar B, Vargas C, Rueda E. Madres descubriendo el amor incondicional en el proceso adaptativo de hospitalización de su bebé prematuro. Rev Cuid [internet]. 2014 [citado 2015 may];5(2):782-9. doi: http://dx.doi.org/10.15649/cuidarte.v5i2.93

17. Cumsille P, Ramírez V. Evaluación de un Programa Comunitario Destinado a Favorecer el Desarrollo Psicosocial de Madres Adolescentes y sus Hijos. Rev Psykhe [internet].2011 [citado 2015 dic];8(2). Disponible en: http://www.psykhe.cl/index.php/psykhe/article/view/131

18. Sánchez Valencia YA, Mendoza Tascón LA, Grisales López MB, Ceballos Molina LY, Bustamente Farfán JC, Castañeda EM, et al. Características poblacionales y factores asociados a embarazo en mujeres adolescentes de Tuluá, Colombia. Rev Chil Obstet Ginecol [internet]. 2013 [citado 2015 dic];78(4):269-81. doi: http://dx.doi.org/10.4067/ S0717-75262013000400005

19. Gómez-Sotelo A, Gutiérrez-Malaver ME, Izzedin-Bouquet $R$, Sánchez-Martínez LM, Herrera-Medina NE, Ballesteros-Cabrera M. Representaciones sociales del embarazo y la maternidad en adolescentes primigestantes y multigestantes en Bogotá. Rev Salud Pública [internet]. 2012 [citado 2015 may];14(2):189-99. Disponible en: http:// www.scielosp.org/pdf/rsap/v14n2/v14n2a01

20. Álvarez Nieto C, Pastor Moreno G, Linares Abad M, Serrano Martos J, Rodríguez Olalla L. Motivaciones para el embarazo adolescente. Gac Sanit [internet]. 2012 [citado 2015 dic];26(6):487-503. Disponible en: http://scielo.isciii.es/scielo.php?script=sci_arttext\&pid=S021391112 012000600002\&lng=es

21. Diniz E, García Días AC, Neiva-Silva L, Nieto CJ, Koller SH. Características familiares y apoyo percibido entre adolescentes brasileños con y sin experiencia de embarazo. Av Psicol Latinoam [internet]. 2012 [citado 2015 abr];30(1):65-80. Disponible en: http://www. redalyc.org/pdf/799/79924085005.pd 
22. Hernández Betancur A. Circunstancias que inducen al embarazo en la adolescencia. Revista Cultura del Cuidado [internet]. 2013 [citado 2015 dic];10(1):27-37. Disponible en: http://repositorio.unilibrepereira. edu.co:8080/pereira/bitstream/handle/123456789/140/Articulo\%20 No\%203\%20Circunstancias\%20que\%20inducen\%20al\%20embarazo. pdf; sequence $=1$

23. Morillo JB, Montero L. Lactancia materna y relación materno filial en madres adolescentes. Enferm Glob [internet]. 2010 [citado 2015 dic];(19). Disponible en: http://scielo.isciii.es/scielo. php?script=sci_arttext\&pid=S1695-61412010000200019\&lng=es

24. Contreras-Pulache H, Mori-Quispe E, Hinostroza-Camposano WD, Yancachajlla-Apaza M, Lam-Figueroa N, Chacón-Torrico H. Características de la violencia durante el embarazo en adolescentes de Lima, Perú. Rev Perú Med Exp Salud Pública [internet]. 2013 [citado 2015 dic];30(3). Disponible en http://www.scielo.org.pe/scielo. php?script $=$ sci_arttext\&pid=S1726-46342013000300002\&lng $=$ es $\&$ nrm=iso

25. Aracena M, Leiva L, Undurraga C, Krause M, Pérez C, Cuadra V, et al. Evaluación de la efectividad de programas de visitas domiciliarias para madres adolescentes y sus hijos/as. Rev Méd Chile [internet]. 2011 [citado 2015 dic];139(1):60-5. doi: http://dx.doi.org/10.4067/ S0034-98872011000100008

26. Gardner M, Deatrick J. Understanding interventions and outcomes in mothers of infants. Issues Compr Pediatr Nurs [internet]. 2006 [citado 2015 feb];29(1):25-44. Disponible en: http://search.ebscohost.com/login. aspx?direct $=$ true $\& \mathrm{db}=\mathrm{pbh} \& \mathrm{AN}=20063402 \&$ site=ehost-live\&scope $=$ site

27. Meleis AI, editor. Becoming a mother versus maternal role attainment. En: Transitions theory: middle range and situation specific theories in nursing research and practice [internet]. New York: Springer; 2010 [citado 2015 mar]. Disponible en: http://taskurun.files.wordpress. com/2011/10/transitions_theory__middle_range_and_situation_specific_theories_in_nursing_research_and_practice.pdf

28. Mercer R. Becoming a mother versus maternal role attainment. JNS [internet]. 2004 [citado 2015 feb];(36):226-7. Disponible en: http://Oproquest.umi.com.millenium.itesm.mx/pqdweb?RQT=318\&p $\mathrm{mid}=37455 \& \mathrm{TS}=1174175249 \&$ clientId=23693\&VType $=$ PQD\&VName $=$ PQD\&VInst=PROD

29. Aracena M, Krause M, Pérez C, Bedral P, Undurraga C, Alamo N. Efectos de mediano plazo de un programa de intervención para madres adolescentes. Atención Primaria [internet]. 2013 [citado 2015 dic];45(3):15764. Disponible en: http://www.sciencedirect.com/science/article/pii/ S0212656712004258

30. Montes M, Argote LA, Álvarez GC, Osorio A, Osorio O. Aplicación de un modelo fundamentado en el amor con adolescentes grávidas en el Centro de Salud Cristóbal Colón, Cali, Colombia. Colombia Médica [internet]. 2005 [citado 2015 dic];36(3):83-92. Disponible en: http://www.google. 
com.co/url?sa=t\&rct=j\&q=\&esrc $=$ s\&source $=$ web\& $c d=1 \& v e d=0 C C O Q F j$ AA\&url=http $\% 3 \mathrm{~A} \% 2 \mathrm{~F} \% 2 \mathrm{Fcolombiamedica.univalle.edu.co \% 2Findex.ph}$ p\%2Fcomedica\%2Farticle\%2Fdownoad\%2F380\%2F384\&ei=Y31wUduS AfOr4APJvYGwBQ\&usg=AFQjCNEaILh1FT3CBALJ31te49VHujPeg\&bv $\mathrm{m}=$ bv.45373924,d.dmg 